\title{
AUTOMORPHISM GROUPS OF ABELIAN $p$-GROUPS
}

\author{
JUTTA HAUSEN 1
}

ABSTRACT. Let $\Gamma$ be the automorphism group of a nonelementary reduced abelian $p$-group, $p \geq 5$. It is shown that every noncentral norinal subgroup of $\Gamma$ contains a noncentral elementary abelian normal $p$-subgroup of $\Gamma$ of rank at least 2 .

1. The result. Throughout the following, $G$ is a reduced $p$-primary abelian group, $p \geq 5$, and $\Gamma$ is the group of all automorphisms of $G$.

If $G$ is elementary abelian then the normal structure of $\Gamma$ is well known. In particular, $\Gamma$ does not contain normal $p$-subgroups $\neq 1[2, \mathrm{pp} .41,45]$. If $p G \neq 0$ then $\Gamma$ does possess nontrivial normal $p$-subgroups. Moreover, in this case, every noncentral (i.e. not contained in the center $Z \Gamma$ of $\Gamma$ ) normal subgroup of $\Gamma$ contains a noncentral normal $p$-subgroup $N$ of $\Gamma$ such that $N^{p}=1[6$, Theorem A].

The purpose of this note is to prove the following result which is considerably stronger.

Theorem. Let $\Gamma$ be the automorphism group of a nonelementary reduced abelian $p$-group, $p \geq 5$. Then every noncentral normal subgroup of $\Gamma$ contains a noncentral elementary abelian normal p-subgroup of $\Gamma$ of rank at least 2 .

The hypothesis $p \neq 2$ is indispensable since the dihedral group $D_{4}$ occurs as an automorphism group of an abelian 2-group (namely $G=Z(2) \oplus$ $Z(4) ; D_{4}$ contains a [noncentral] cyclic normal subgroup of order 4 whose socle is the center of $\left.D_{4}\right)$. Whether the above Theorem holds true for $p=3$ is an open question.

2. Tools. Notation and terminology will be that of [3] and [6] unless explained otherwise. Calculations involving automorphisms are carried out in the endomorphism ring of $G$. The following facts are used frequently. Note that mappings are written to the right.

Received by the editors December 26, 1973.

AMS (MOS) subject classifications (1970)。Primary $20 \mathrm{~K} 30,20 \mathrm{~K} 10,20 \mathrm{~F} 15$; Secondary $20 \mathrm{~F} 30$.

Key words and phrases. Abelian p-group, automorphism group, normal subgroups of automorphism groups.

${ }^{1}$ This research was supported in part by the National Science Foundation under Grant GP-34195. 
(2.1) The center of $\Gamma$. The center of $\Gamma$ consists precisely of the multiplications with units in the ring $R_{p}$ of $p$-adic integers $[1, \mathrm{pp} .110,111]$. If $G$ is unbounded then $Z \Gamma \simeq R_{p}^{*}$ and the center of $\Gamma$ contains no elements of order $p$. An automorphism $\alpha$ of $G$ is central if and only if $S \alpha=S$ for all subgroups $S$ of $G$ [1, pp. 110,111]. $\Gamma$ is commutative if and only if $G$ is (locally) cyclic [3, p. 222].

(2.2) Stabilizers. Let fix $(B / A)$ be the set of all $\gamma \in \Gamma$ inducing the identity mapping in $B / A$ where $A \leq B$ are subgroups of $G$. If $A$ and $B$ are characteristic in $G$ then $\operatorname{fix}(B / A)$ is a normal subgroup of $\Gamma$. The stabilizer of $A$ in $G$ is defined as stab $A=$ fix $A \cap$ fix $(G / A)$. It is well known that stab $A \simeq \operatorname{Hom}(G / A, A)$. In particular, stabilizers are abelian.

(2.3) The normal subgroups of exponent $p$. Let $N$ be a normal subgroup of $\Gamma$ such that $N^{p}=1$. Then $N \leq \Psi$ where $\Psi$ consists of all $\gamma \in \Gamma$ such that $G[p](\gamma-1) \leq p G$ and $p(\gamma-1)=0[4$, pp. 409, 410]. If $\psi \in \Psi$ then $G(\psi-1) \leq G[p]$ and $(\psi-1)^{3}=0[4$, p. 411]. Hence $\Psi \leq$ fix $(p G) \cap$ fix $(G / G[p])$ and, since $p \geq 3, \Psi^{p}=1$.

An immediate consequence is the following result.

Lemma 2.4. If $N$ is a normal p-subgroup of $\Gamma$ such that $N^{p}=1$ then $N \cap$ fix $G[p]$ and $N \cap \operatorname{fix}(G / p G)$ are elementary abelian normal p-subgroups of $\Gamma$.

Proof. By (2.3) $N \leq \Psi$, and $\Psi \cap$ fix $G[p] \leq \operatorname{stab} G[p], \Psi \cap$ fix $(G / p G) \leq$ stab $p G$. By (2.2)

$\operatorname{stab} G[p] \simeq \operatorname{Hom}(G / G[p], G[p]), \quad \operatorname{stab} p G \simeq \operatorname{Hom}(G / p G, p G)$, which are elementary abelian.

The following two lemmas are technical.

Lemma 2.5. Let $G=A \oplus\langle h\rangle$ where $A$ has rank at least two and $p^{m} A=$ $0 \neq p^{m} G$ for some integer $m \geq 1$. Let $N$ be a normal subgroup of $\Gamma$ such that $N^{p}=1$. If there exists $\gamma \in N$ such that $(y-1)^{2} \neq 0$ then $N \cap$ fix $G[p]$ is noncentral.

Proof. Let $\gamma=1+\tau \in N$ such that $\tau^{2} \neq 0$. Then $G \tau \leq G[p], p \tau=0$ (cf. (2.3)), and $r^{2} \neq 0$ implies $G \tau \not \subset p G$. Since $G$ is generated by its elements of maximal order, one can assume $h r^{2} \neq 0$. Hence $h r \in G[p] \backslash p G$ and

$$
G=\langle a\rangle \oplus B \oplus\langle b\rangle, \quad b r=a, \quad a r \neq 0, \quad B \neq 0 .
$$

Suppose $p B=0$. Then $p G=\langle p h\rangle$ and $\left\langle p^{m} h\right\rangle \geq(p G)[p] \geq G[p] \tau \geq(\langle a\rangle \oplus B) \tau$. Hence $a r \neq 0$ and $B \neq 0$ imply 
(a) $\oplus B=\langle a\rangle \oplus K, \quad K \tau=0 \neq K$.

In this case, pick any $0 \neq x \in K$. If $p B \neq 0$ pick $0 \neq x \in(p B)[p]$ and put $K=B$. In either case

$$
G=\langle a\rangle \oplus K \oplus\langle h\rangle, \quad 0 \neq x \in K[p], \quad x \tau=0 .
$$

Define the endomorphism $\sigma$ of $G$ by

$$
a \sigma=x, \quad K \sigma=0, \quad h \sigma=0 .
$$

Then $\sigma^{2}=0$ and $G \sigma \tau=\langle x\rangle \tau=0$. Lemma 2.6 of [7] implies $\delta=1+\tau \sigma \in N$. From $h(\delta-1)=h r \sigma=a \sigma=x \notin\langle h\rangle$ it follows that $\delta \notin Z \Gamma$ (cf. (2.1)). Since

$$
G[p](\delta-1)=G[p] \tau \sigma \leq p G \sigma=p \cdot\langle x\rangle=0,
$$

$\delta \in N \cap$ fix $G[p]$, completing the proof.

Lemma 2.6. Let $G$ and $N$ be as in Lemma 2.5 and suppose that $(\gamma-1)^{2}$ $=0$ for all $\gamma \in N$. If $N \cap$ fix $G[p] \leq Z \Gamma$ and $N \cap \operatorname{fix}(G / p G) \leq Z \Gamma$ then $N \leq Z \Gamma$.

Proof. Assume by way of contradiction that there exists $y=1+\tau \epsilon$ $N \backslash Z \Gamma$. Then $\gamma \notin \mathrm{fix}(G / p G)$ and, as above, one can assume $h r \notin p G$, consequently $h r \in G[p] \backslash p G$ and

$$
G=\langle a\rangle \oplus B \oplus\langle b\rangle, \quad h r=a, \quad O(a)=p, \quad a \tau=h r^{2}=0 .
$$

By hypothesis $\gamma \notin$ fix $G[p]$ and hence $G[p] \tau \neq 0$. Since $G[p] \leq\langle a\rangle \oplus B[p]$ $\oplus\langle p h\rangle$ and $a \tau=0, p \tau=0$, this implies the existence of $b \in B[p] \backslash p B$ such that

$$
b r \neq 0
$$

Let $k=2^{-1}(p+1)$. Since $p$ is odd, $k$ is an integer and $k$ and $p$ are relatively prime. Using (2.7), define $\beta \in \Gamma$ by

$$
a \beta=k a+b, \quad(B \oplus\langle h\rangle)(\beta-1)=0 .
$$

Note that $b r \beta=b \tau$ since $b \tau \in p G \leq B \oplus\langle h\rangle$. One verifies that $a \beta^{-1}=$ $2(a-b)$, and hence

$$
\begin{aligned}
& a \beta^{-1} \tau \beta=2(a-b) \tau \beta=-2 b \tau \beta=-2 b \tau, \\
& b \beta^{-1} \tau \beta=b \tau \beta=b \tau, \\
& b \beta^{-1} \tau \beta=b \tau \beta=a \beta=k a+b .
\end{aligned}
$$


Let $\delta=\gamma \beta^{-1} \gamma \beta=(1+\tau)\left(1+\beta^{-1} \tau \beta\right)$. Then $\delta \in N$ and $\delta=1+\eta$, where $\eta=\tau+\beta^{-1} \tau \beta+\tau \beta^{-1} \tau \beta$. Since

$$
\begin{aligned}
h(\delta-1) & =h \eta=h \tau+h \beta^{-1} \tau \beta+h \tau \beta^{-1} \tau \beta \\
& =a+(k a+b)+(-2 b \tau)=(k+1) a+b-2 b \tau
\end{aligned}
$$

and $b r \in p G$, it follows that $h(\delta-1) \notin\langle h\rangle$. Hence $\delta \in N$ is noncentral (cf. (2.1)) and, by hypothesis, $(\delta-1)^{2}=0$. From $a, b \in G[p], G[p] \tau \beta^{-1} \tau \beta \leq$ $p G \beta^{-1} \tau \beta=p G \tau \beta=0$, and $\tau^{2}=0$, one obtains

$$
\begin{aligned}
0 & =b(\delta-1)^{2}=[(k+1) a+b-2 b \tau] \eta \\
& =[(k+1) a+b-2 b \tau]\left(\tau+\beta^{-1} \tau \beta+\tau \beta^{-1} \tau \beta\right) \\
& =[(k+1) a+b]\left(\tau+\beta^{-1} \tau \beta\right)=(k+1) a\left(\tau+\beta^{-1} \tau \beta\right)+b\left(\tau+\beta^{-1} \tau \beta\right) \\
& =(k+1)(-2 b \tau)+b \tau+b \tau \\
& =-2 k b \tau=-2 \cdot 2^{-1}(p+1) b \tau=-b \tau .
\end{aligned}
$$

Hence $b \tau=0$, contradicting (2.8) and proving the lemma.

Corollary 2.9. Let $G$ and $N$ be as in Lemma 2.5. If $N$ is noncentral then $N \cap$ fix $G[p]$ or $N \cap \operatorname{fix}(G / p G)$ is noncentral.

3. Proof. Assume the situation of the Theorem and let $N$ be a noncentral normal subgroup of $\Gamma$. It was shown in [6] that cyclic normal subgroups of $\Gamma$ are central. Hence, it suffices to show that $N$ contains a noncentral elementary abelian normal $p$-subgroup of $\Gamma$. By Theorem $A$ of [6], every noncentral normal subgroup of $\Gamma$ contains a noncentral normal subgroup of $\Gamma$ of exponent $p$. This permits the assumption

$$
N^{p}=1 \text {. }
$$

Distinguish the following cases.

Case 1. $G$ is unbounded. Then $Z \Gamma$ contains no elements of order $p$ (cf. (2.1)) and every $p$-subgroup $\neq 1$ of $\Gamma$ is noncentral. Therefore, using (3.1) and Lemma 2.4, it suffices to prove $N \cap$ fix $G[p] \neq 1$. Let, for $n \geq 1$ an integer, $\Sigma_{n}=$ stab $G\left[p^{n}\right]$. Then $\Sigma_{n} \leq$ fix $G[p]$ and the proof will be completed by showing $N \cap \Sigma_{n} \neq 1$ for some $n$. Assume, by way of contradiction, that $N \cap \Sigma_{n}=1$ for all $n \geq 1$. Since $N$ and the $\Sigma_{n}$ are normal subgroups of $\Gamma$ this implies $N$ is contained in the centralizer $C \Sigma_{n}$ of $\Sigma_{n}$ in $\Gamma$, for all $n \geq 1$. By Lemma 2.1 of $[5], C \Sigma_{n} \leq Z \Gamma \cdot$ fix $G\left[p^{n}\right]$. Hence 


$$
N \leq \bigcap_{n \geq 1} C \Sigma_{n} \leq \bigcap_{n \geq 1}\left(Z \Gamma \cdot \text { fix } G\left[p^{n}\right]\right)=\Phi .
$$

Using (2.1) and the fact that $G=\bigcup_{n \geq 1} G\left[p^{n}\right]$, one verifies that every $\phi \in \Phi$ induces the identity mapping in the lattice of all subgroups of $G$ and hence, $\phi \in Z \Gamma$. This together with (3.2) implies $N \leq Z \Gamma$ which is the desired contradiction.

Case 2. $G$ is bounded. It has been proved in [7] that $G$ is a bounded group with two independent elements of maximal order if and only if the intersection $D \Gamma$ of all noncentral normal subgroups of $\Gamma$ is noncentral; and $D \Gamma$ is an elementary abelian $p$-group if $p G \neq 0[7$, Theorems 2,3$]$. Therefore, one can assume that $G=A \oplus\langle h\rangle$ and $p^{m} G \neq 0=p^{m} A$ for some integer $m \geq 1$. If $A$ has rank at least 2, Corollary 2.9, Lemma 2.4, and (3.1) finish the proof. Suppose that

$$
G=\langle a\rangle \oplus\langle b\rangle, \quad O(a)<O(b)=p^{m+1} .
$$

If $p a \neq 0$ then $G[p] \leq p G$ and (3.1), (2.3), and (2.2) imply

$$
N \leq \Psi \leq \operatorname{stab} G[p] \simeq \operatorname{Hom}(G / G[p], G[p]) .
$$

Hence $N$ is elementary abelian and the proof is completed.

It remains to consider the case where $a$ in (3.3) has order p. By (3.1) and (2.3), $N \leq \Psi$ and the elements in $\Psi$ can be identified with matrices of the form

$$
\left[\begin{array}{cc}
1 & p^{m} k \\
l & 1+p^{m} n
\end{array}\right]
$$

where $0 \leq k, l, n \leq p-1$ are integers. Hence $\Psi$ has order $p^{3}$ and either $N=\Psi$ or $N$ has order $p$ or $p^{2}$. If $N=\Psi$ then $N$ contains noncentral elementary abelian normal $p$-subgroups of $\Gamma$, for instance stab $G[p] \leq \Psi$. If $N$ has order $p^{2}$ or $p$, then $N$ is commutative and, because of (3.1), $N$ is elementary abelian. (Actually, the case $|N|=p$ cannot occur since cyclic normal subgroups of $\Gamma$ are contained in $Z \Gamma[6$, Theorem $B]$.) The Theorem is proven.

\section{REFERENCES}

1. R. Baer, Primary abelian groups and their automorphisms, Amer. J. Math. 59 (1937), 99-117.

2. J. Dieudonné, Les déterminants sur un corps non commutatif, Bull. Soc. Math. France 71 (1943), 27-45. MR 7, 3.

3. L. Fuchs, Abelian groups, Akad. Kiadó, Budapest, 1958; republished by Internat. Ser. Monographs on Pure and Appl. Math., Pergamon Press, New York, 1960. MR 21\#5672; $22 \# 2644$. 
4. J. Hausen, On the normal structure of automorphism groups of abelian p-groups, J. London Math. Soc. (2) 5 (1972), 409-413.

5. - The automorphism group of an abelian p-group and its noncentral normal subgroups, J. Algebra 30 (1974), 459-472.

6. - The automorphism group of an abelian p-group and its normal p-subgroups, Trans. Amer. Math. Soc. 182 (1973), 159-164.

7. - Structural relations between general linear groups and automorphism groups of abelian p-groups, Proc. London Math. Soc. (3) 28 (1974), 614-630.

DEPARTMENT OF MATHEMATICS, UNIVERSITY OF HOUSTON, HOUSTON, TEXAS 77004 